\title{
Variability Time Scales of GRBs
}

\section{G. A. MacLachlan*}

The George Washington University, Department of Physics

E-mail: maclach@gwu. edu

\section{A. Shenoy ${ }^{a}$, E. Sonbas ${ }^{b}$, K. S. Dhuga ${ }^{a}$, W. C. Parke ${ }^{a}$, L. C. Maximon ${ }^{a}$, A.}

\section{Eskandarian $^{a}$}

${ }^{a}$ Department of Physics, The George Washington University

Washington, DC 20052

${ }^{b}$ University of Adiyaman, Department of Physics,

02040, Adiyaman, Turkey

We have investigated the time variations in the light curves from a sample of long and short Fermi/GBM Gamma ray bursts (GRBs) using an impartial wavelet analysis and compared those results with results from a pulse fitting analysis. We present a direct link between the minimum variability time scales extracted through the wavelet decomposition and the rise times of the shortest pulses extracted via the pulse fits of light curves. The results indicate minimum variabilities from a few milliseconds to several hundred milliseconds are not uncommon.

Gamma-Ray Bursts 2012 Conference-GRB2012,

May 07-11, 2012

Munich, Germany

\footnotetext{
* Speaker.
} 


\section{Introduction}

The prompt emission from Gamma-ray Bursts (GRBs) shows complex time profiles that have eluded a generally accepted explanation. Several models have been proposed to explain the observed temporal variability of GRB light curves. Leading models such as the internal shock model [3] and the photospheric model [10] link the rapid variability directly to the activity of the central engine. Others invoke relativistic outflow mechanisms to suggest that local turbulence amplified through Lorentz boosting leads to causally disconnected regions which in turn act as independent centers for the observed prompt emission. Within more recent models, both [6] and [11] argue that the temporal variability may show two different scales depending on the physical mechanisms generating the prompt emission.

One approach for probing light curves for temporal structures which has received attention $[7,9,2,8]$ is to express them as a series of displaced pulses, each with a parametric form. There is an appeal to this approach because fitting routines are well-understood and interpretations of rise time, decay time, full width at half max, etc, are possible. On the other hand, one must make certain assumptions when using the pulse-fitting procedure such as the choice of the functional form to use for an individual pulse and the number of parameters to be included in the fitting function. Moreover, light with high variability at low power may show variations which are not statistically significant.

A complementary approach using a wavelet-based analysis of a set of both long and short GRB light curves is discussed by [4] in which a time scale, $\tau_{\beta}$, is identified that marks the transition from white noise to a power law in the power density spectrum (a $f^{-\alpha}$ behavior). It is argued that over time scales smaller than $\tau_{\beta}$ the light curves appear stochastic and signal power is distributed uniformly. At time scales larger than $\tau_{\beta}$, identifiable structures (such as pulses) with signal power are no longer distributed uniformly over the periods of light variation. For this reason $\tau_{\beta}$ is referred to as the minimum variability time scale.

The analysis presented in [4] is a non-parametric approach to probing light curves for time scales. It makes no assumptions about the nature of the structures in a given light curve that give rise to the $f^{-\alpha}$ character. The technique, however, offers no firm connection between $\tau_{\beta}$ and the constituent structures although it seems reasonable to associate $\tau_{\beta}$ with the scale of the smallest emitting structures present.

Results from an application of a log-normal pulse-fitting procedure to GRB light curves have been published by [1]. In this paper we make a meta-analysis of the timing results presented by [1] compared with the techniques of [4] for a set of 34 GRBs used in both studies.

\section{Analysis}

We begin by considering the relation between $\tau_{\beta}$ and the pulse parameters given in Table 3 of [1]. The parameters with temporal units in Table 3 are: time-since-trigger, rise time, decay time, and FWHM. In all, 34 GRBs comprising 379 pulses are considered here although we focus only on the shortest pulse per GRB.

The most likely candidates for producing a minimum variability time scale are the shortest pulses and in Fig. 1 we plot only the smallest rise times for each GRB against $\tau_{\beta}$. We argue that [1] 


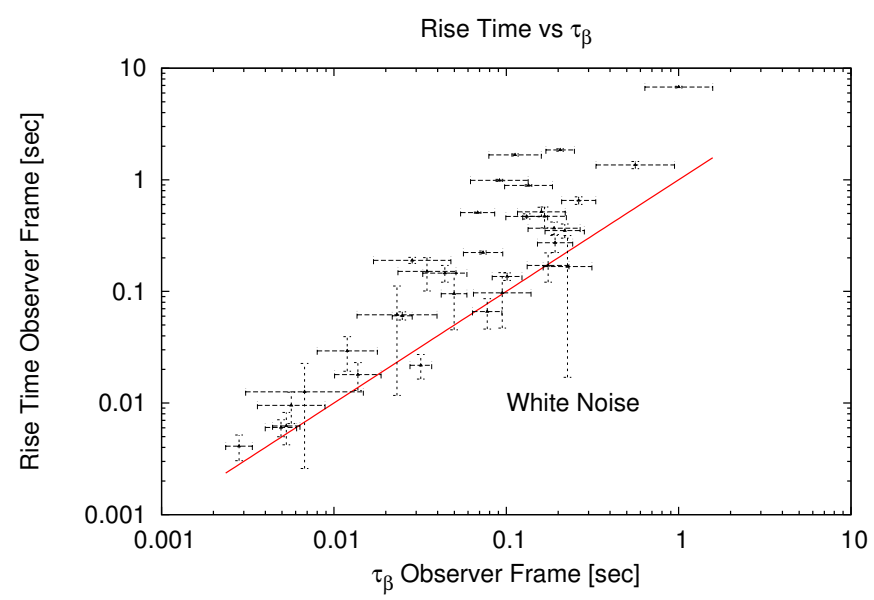

Figure 1: Minimum variability time scale versus rise time in the Observer frame with only smallest rise times included. The line represents the locus where $\tau_{\beta}=$ rise time. We identify the area below the line with white noise. The data are expected to press up against the line from above but not to cross it. This plot gives substance to the interpretation of the minimum variability time scale.

and [4] have tracked the same physical observables over approximately three orders of magnitude using independent methods.

An equality line is also shown which is the locus where $\tau_{\beta}$ equals rise time. We argue as in [5] that $\tau_{\beta}$ represents the minimum variability time scale and the space in the $\tau_{\beta}$-rise time plane below the equality line should be interpreted as a structureless white noise region.

\section{Results and Discussion}

For a large sample of short and long Fermi GBM bursts, [4] used a technique based on wavelets to determine the minimum variability time scale, $\tau_{\beta}$. The authors associate this time scale with a transition from red-noise processes to parts of the power spectrum dominated by white noise or random noise components. Accordingly, the authors note that this time scale is the shortest resolvable variability time for physical processes intrinsic to the GRB. In addition, histograms of the values of $\tau_{\beta}$ for long and short GRBs were shown to exhibit a clear temporal offset in the mean $\tau_{\beta}$ values for long and short GRBs.

In a separate analysis, using a particular functional form for pulse shapes, [1] have extracted an extensive set of key pulse parameters such as rise times, decay times, widths (FWHM), and times since trigger for a host of bright GRBs detected by Fermi/GBM. Using the FWHM values, these authors also reported a significant temporal offset between the mean values for long and short GRBs.

\section{CONCLUSIONS}

In a meta-analysis of results by [1] and by [4], we have studied the relationship between key parameters that describe the temporal properties of a sample of prompt-emission light curves for 
long and short-duration GRBs detected by the Fermi/GBM mission. We compare the minimum variability timescale extracted through a technique based on wavelets, with the pulse-time parameters extracted through a pulse-fitting procedure. Our main results are summarized as follows:

a) Both methods indicate a temporal offset between short and long-duration bursts. The quantitative agreement between the two methods is quite good.

b) Both methods point to scaling trends between characteristic timescales. In the case of the pulse-fitting method the scaling appears to involve parameters such as rise times, FWHM, and pulse intervals. For the wavelet technique, the scaling involves a correlation between the minimum variability time scale and the duration of the bursts.

c) By demonstrating a strong positive correlation between $\tau_{\beta}$ and the rise time of the shortest fitted pulses, we provide for the first time, a direct link between the shortest resolvable temporal structure in a GRB lightcurve with that of a key pulse profile parameter.

d) By combining the two techniques, we have shown that one can arrive at a much tighter demarcation of the boundary between the power spectrum domains that separate red noise and white noise processes.

\section{References}

[1] P.N. Bhat, et al. Temporal Deconvolution Study of Long and Short Gamma-Ray Burst Light Curves, ApJ 744 (2012) 141 (online-version)

[2] J. Hakkila, \& R. J. Nemiroff, Testing The Gamma-Ray Burst Pulse Start Conjecture, ApJ 705 (2009) 372

[3] S. Kobayashi, T. Piran, \& R. Sari, Can Internal Shocks Produce the Variability in Gamma-Ray Bursts?, ApJ 490 (1997) 92

[4] G.A. MacLachlan, et al. Minimum Variability Time Scales of Long and Short GRBs (2012) [arXiv:1201.4431v2]

[5] G.A. MacLachlan, G. A., The Minimum Variability Time Scale and its Relation to Pulse Profiles of Fermi GRBs (2012) [arXiv: 1205 .0055] (accepted by MNRAS)

[6] B. J. Morsony, D. Lazzati \& M. C. Begelman, The origin and propagation of variability in the outflows of long duration gamma-ray bursts (2010) [arXiv: 1002.0361]

[7] R. J. Nemiroff, et al. The Pulse Scale Conjecture and the Case of BATSE Trigger 2193, ApJ 544 (2000) 805

[8] R. J. Nemiroff, Extension of an exponential light-curve gamma-ray burst pulse model across energy bands, MNRAS 419 (2012) 1650

[9] J. P. Norris et al. Long-Lag, Wide-Pulse Gamma-Ray Bursts, ApJ 627 (2005) 324

[10] F. Ryde, The Cooling Behavior of Thermal Pulses in Gamma-Ray Bursts, ApJ 614 (2004) 827

[11] B. Zhang, \& H. Yan, The Internal-Collision-Induced Magnetic Reconnection and Turbulence (ICMART) Model of Gamma-Ray Bursts, ApJ 726 (2011) 90 